\title{
Polymer microarrays rapidly identify competitive adsorbents of virus-like particles
}

(F)

Cite as: Biointerphases 15, 061005 (2020); https://doi.org/10.1116/6.0000586

Submitted: 27 August 2020 . Accepted: 15 October 2020 . Published Online: 17 November 2020

(D) Andrew J. Blok, (D) Pratik Gurnani, Alex Xenopoulos, (D) Laurence Burroughs, (D) Joshua Duncan, (D) Richard A. Urbanowicz, (D) Theocharis Tsoleridis, Helena Müller-Kräuter, Thomas Strecker, (D) Jonathan K. Ball, (D) Cameron Alexander, and (D) Morgan R. Alexander

\section{COLLECTIONS}

F This paper was selected as Featured
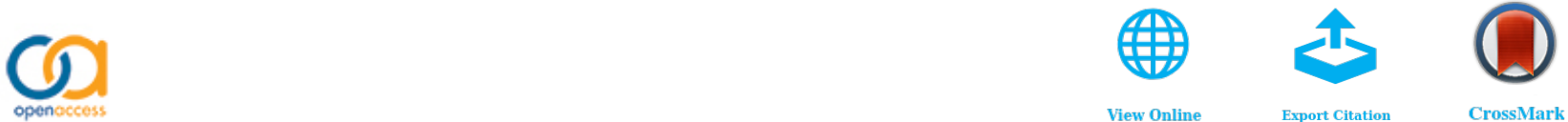

\section{ARTICLES YOU MAY BE INTERESTED IN}

Extremal quantum states

AVS Quantum Science 2, 044701 (2020); https://doi.org/10.1116/5.0025819

Cold atmospheric plasma for SARS-CoV-2 inactivation

Physics of Fluids 32, 111702 (2020); https://doi.org/10.1063/5.0031332

Travel restrictions during pandemics: A useful strategy?

Chaos: An Interdisciplinary Journal of Nonlinear Science 30, 111103 (2020); https://

doi.org/10.1063/5.0028091

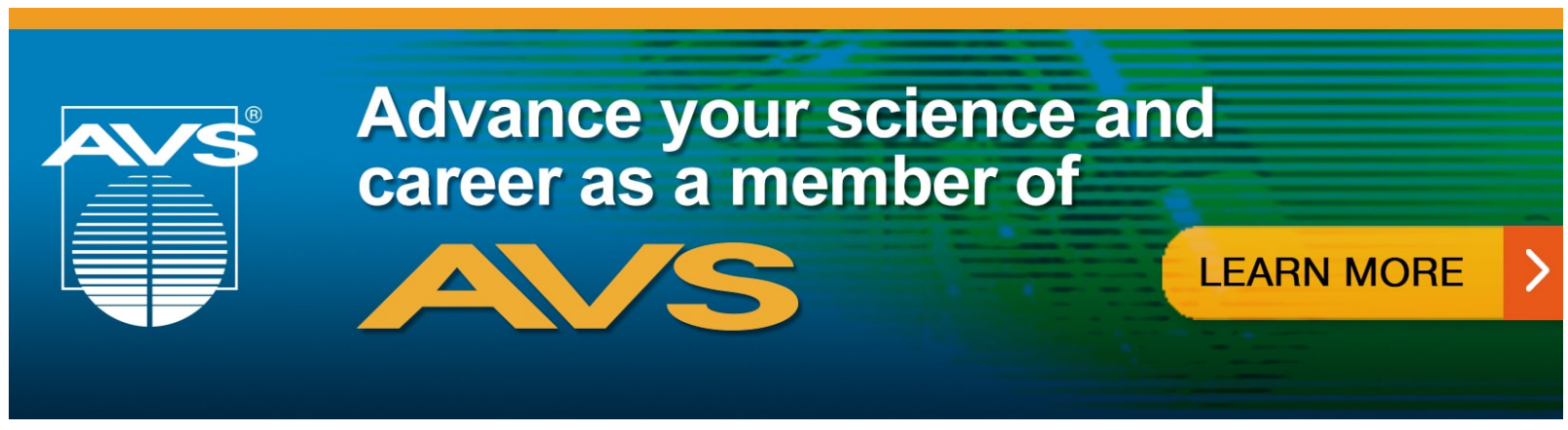




\title{
Polymer microarrays rapidly identify competitive adsorbents of virus-like particles
}

\author{
Cite as: Biointerphases 15, 061005 (2020); doi: $10.1116 / 6.0000586$ \\ Submitted: 27 August 2020 . Accepted: 15 October 2020 . \\ Published Online: 17 November 2020
}

\author{
Andrew J. Blok, ${ }^{7}$ (D) Pratik Gurnani, ${ }^{1}$ (D) Alex Xenopoulos, ${ }^{2}$ Laurence Burroughs, ${ }^{3}$ (D) Joshua Duncan, ${ }^{4,5}$ (iD \\ Richard A. Urbanowicz, ${ }^{4,5}$ (D) Theocharis Tsoleridis, ${ }^{4,5}$ (D) Helena Müller-Kräuter, ${ }^{6}$ Thomas Strecker, $^{6}$ \\ Jonathan K. Ball, ${ }^{4,5}$ (D) Cameron Alexander, ${ }^{7}$ (D) and Morgan R. Alexander ${ }^{3}$ (D) \\ AFFILIATIONS \\ ${ }^{1}$ Division of Molecular Therapeutics and Formulation, School of Pharmacy, University of Nottingham, Nottingham NG7 2RD, \\ United Kingdom \\ EMD Millipore, 80 Ashby Road, Bedford, Massachusetts 01730 \\ ${ }^{3}$ Division of Advanced Materials and Healthcare Technologies, School of Pharmacy, University of Nottingham, \\ Nottingham NG7 2RD, United Kingdom \\ ${ }^{4}$ Wolfson Centre for Global Virus Research, Faculty of Medicine and Health Science, University of Nottingham, \\ Nottingham NG7 2RD, United Kingdom \\ ${ }^{5}$ Nottingham Biomedical Research Centre, C Floor, South Block, Queen's Medical Centre, Nottingham NG7 2UH, \\ United Kingdom \\ ${ }^{6}$ Institute of Virology, Philipps University of Marburg, Marburg 35043, Germany
}

\begin{abstract}
The emergence of SARS-CoV-2 highlights the global need for platform technologies to enable the rapid development of diagnostics, vaccines, treatments, and personal protective equipment (PPE). However, many current technologies require the detailed mechanistic knowledge of specific material-virion interactions before they can be employed, for example, to aid in the purification of vaccine components or in the design of a more effective PPE. Here, we show that an adaption of a polymer microarray method for screening bacterial-surface interactions allows for the screening of polymers for desirable material-virion interactions. Nonpathogenic virus-like particles including fluorophores are exposed to the arrays in an aqueous buffer as a simple model of virions carried to the surface in saliva/sputum. Competitive binding of Lassa and Rubella virus-like particles is measured to probe the relative binding properties of a selection of copolymers. This provides the first step in the development of a method for the discovery of novel materials with promise for viral binding, with the next being development of this method to assess absolute viral adsorption and assessment of the attenuation of the activity of live virus, which we propose would be part of a material scale up step carried out in high containment facilities, alongside the use of more complex media to represent biological fluids.
\end{abstract}

(C) 2020 Author(s). All article content, except where otherwise noted, is licensed under a Creative Commons Attribution (CC BY) license (http://creativecommons.org/licenses/by/4.0/). https://doi.org/10.1116/6.0000586

\section{INTRODUCTION}

Common strategies for selective biomolecular recognition in diagnostics typically utilize antigen-antibody interactions, such as in common ELISA immunoassays. ${ }^{1,2}$ While these assays typically allow high selectivity to be obtained, there are a number of drawbacks that limit their more widespread usage, including the cost of manufacture (each antigen needs a specific antibody to be developed) and the storage and transport of what are typically thermally sensitive reagents. These disadvantages become more important when the target application requires interactions with classes of related biomolecules rather than specific individual analytes. Prior studies have used low-cost polymers to modify nanocrystals ${ }^{3}$ and chromatographic materials ${ }^{4,5}$ with the aim of introducing broadspectrum binding affinity toward viral targets. However, the myriad of putative copolymer structures derived from even a small number of monomers means that to date only a fraction of the chemical space available for polymeric affinity agents and biomolecular sequestrants has been explored.

Polymer microarrays have been developed to facilitate simultaneous investigation of many thousands of chemically unique materials 
for biologic-material affinity on a single surface. ${ }^{6-13}$ This highthroughput approach has now been used to identify materials for a range of biomedical applications, such as the inhibition of bacterial biofilm formation ${ }^{13}$ and the growth of stem cells with controllable behavior. ${ }^{8}$ Polymer microarrays can be easily fabricated using inkjet or contact printing, coupled with in situ polymerization from low quantities of commercial photocurable monomers.

In this work, we present a method based on a polymer microarray platform to rapidly identify materials derived from commercially available monomers capable of differential adsorption of virus-like particles (VLPs) in competitive binding experiments. The fabricated array contained monomer units that were expected to interact with viral surface components via charge-mediated association and incorporated additional hydrophobic and hydrophilic comonomers to tune relative binding affinities. The method was exemplified using nonreplicating VLPs, from Rubella and Lassa viruses, as structural mimics of infectious disease-causing pathogens but without the full genome encoding for infectivity. These are ideally suited to probing viral binding outside the high level of biosafety restrictions required for live-virus work.

\section{EXPERIMENTAL}

Various approaches used to fabricate polymer microarrays with in situ polymerization onto hydrogel coated glass slides have been previously described. ${ }^{14-17}$ The polymer microarray in this study involved contact printing and subsequent in situ photopolymerization [Fig. 1(a)] of an array of 300 monomer mixtures (14 homopolymers, alongside 182 binary, 72 ternary, and 32 quaternary copolymers) onto an epoxy functionalized, poly (2-hydroxyethylmethacrylate) (pHEMA) coated glass slide in triplicate (the pHEMA coating preventing fouling of the background array surface). Further details of the slide preparation and details on the identity of the 300 copolymers (Tables S1 and S2) are included in the supplementary material. ${ }^{21}$ In brief, the 300 copolymers were formed from the 14 monomers depicted in Fig. 1(b). The charged monomers were selected to provide electrostatic interactions with charged amino acid residues in the surface-displayed VLP proteins. The other monomers (hydrophilic and hydrophobic) were selected to broaden the chemical diversity of the array system and introduce further selectivity via $\mathrm{H}$-bonding interactions at, for example, serine and threonine residues or solvophobic association at leucine, isoleucine, and aromatic rich regions of the proteins and at viral lipid membranes and to potentially reduce protein adsorption in complex biological matrices. After printing, the fabricated slides were imaged using phase contrast microscopy [Fig. S2(a)], and the chemical identities of the polymer spots were analyzed using time of flight-secondary ion mass spectrometry (ToF-SIMS); the representative ion image is presented in Fig. S2(b). Both techniques showed good polymer spot localization, and evidence from ToF-SIMS indicated no significant carryover between the printing of different monomer solutions after appropriate optimization of the process parameters.

We then assessed the short-term adsorption, as an accessible measure for binding interactions, of each VLP to the library in aqueous media as a simplified version of biological fluids. A solution containing both AlexaFluor-488 tagged Rubella VLPs
$(2.5 \mu \mathrm{g} / \mathrm{ml})$ and AlexaFluor-555 tagged Lassa VLPs $(2.5 \mu \mathrm{g} / \mathrm{ml})$ in Dulbecco's phosphate buffered saline (DPBS) was prepared and incubated with the polymer arrays (details of the VLP preparation are included in the supplementary material). ${ }^{21}$ The concentrations chosen were arbitrary, and it is anticipated that to fully understand binding, a range of concentrations would be employed in future work. The microarray was washed $(4 \times 100 \mathrm{ml}$ Milli-Q water, $1 \times 100 \mathrm{ml} \mathrm{DPBS}$ ) and then placed immediately into the incubation solution and rocked in the dark gently at ambient temperature to achieve uniform exposure of the spots to the VLPs. After $4 \mathrm{~h}$ (a time found to be optimal for measuring equilibrium adsorption when investigating proteins), the slide was washed again $(1 \times 100 \mathrm{ml}$ DPBS, $4 \times 100 \mathrm{ml}$ Milli-Q water $)$ in order to remove poorly adhered materials. Fluorescence images of the array both before and after incubation were acquired using an automated microscope (IMSTAR) and processed using IMAGEJ software. Each spot of a composite image (autofluorescence was subtracted from the final result) was cropped using a circle to the border of the spots in order to determine the fluorescence intensity per pixel from each spot due to VLP binding.

\section{RESULTS AND DISCUSSION}

After incubation with dye-labeled VLPs, there was measurable fluorescence intensity (at least $3 \times$ background) observed on the array slide, localized on some of the printed spots, indicating adsorption [Fig. 1(c), Fig. S3 in the supplementary material shows there was little autofluorescence]. ${ }^{21}$ The results were broadly reproducible even with nonoptimized incubation protocols, with the three technical repeats for both types of VLPs showing very similar fluorescence profiles. It was also apparent that preferential adsorption of one labeled VLP compared to the other occurred at some polymer spots. To gain a measure of this selectivity, a binding selectivity index of the fluorescence intensity response per polymer spot pixel for Lassa over Rubella was calculated and is shown for selected polymers in Fig. 2. This approach enabled the rapid identification of copolymer materials that were capable of discriminating between different VLPs based on competitive adsorption. Furthermore, it was possible to separate the selectivity ratios into statistically significant groups $(\mathrm{p}<0.05)$ for high, intermediate, and low VLP-binding polymers (Fig. 2, Fig. S4, Table S3). ${ }^{21}$ These data provide insight into the chemistries of copolymers able to sequester Lassa and/or Rubella VLPs from aqueous suspensions.

The copolymers were chosen to be combinations of monomers with ionizable functionality to interact with proteins on an electrostatic basis and with monomers containing noncharged hydrophilic and hydrophobic side-chains to modulate H-bonding and hydrophobic associations at noncharged regions of VLP surfaces. The highest selectivity ratio of Lassa over Rubella was shown for a copolymer produced from a monomer mixture 66:17:17 tBAEMA:HEMA:MAAcid \% w/w (see Fig. 2 for structures, Fig. S4, Table S3). ${ }^{21}$ All of the top Lassa/Rubella VLP binders contained significant amounts (>30\%) of monomers expected to be positively charged under the incubation conditions (DPBS buffer). The Lassa VLPs contain a tripartite spike complex derived from a single precursor glycoprotein, ${ }^{18}$ whose computational studies have predicted to display an isoelectric point of 7.54 . $^{19}$ The protein part of the 


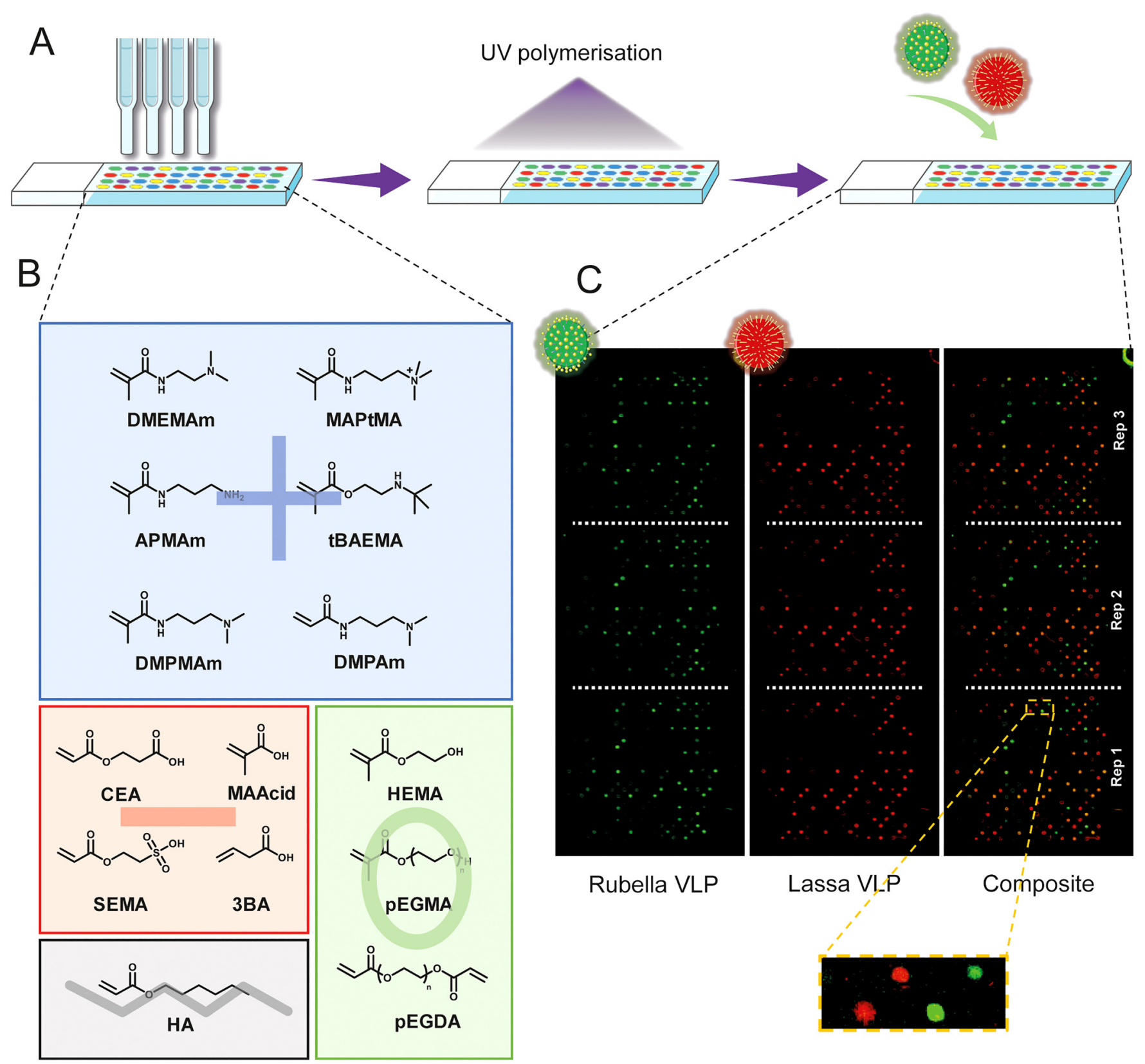

FIG. 1. (a) Schematic depiction of the material identification process, showing the contact printing of the monomers, their in situ UV polymerization, and array incubation with differently fluorescently tagged virus-like particles, (b) the group of monomers used to create the 300 unique copolymers on the array, and (c) composite fluorescence microscopy images (without background subtraction) of the fabricated array slides incubated with $2.5 \mu \mathrm{g} / \mathrm{ml}$ AlexaFluor488 tagged Rubella VLPs and $2.5 \mu \mathrm{g} / \mathrm{ml}$ AlexaFluor555 tagged Lassa VLPs in DPBS buffer for $4 \mathrm{~h}$ at room temperature. The inset shows a zoomed in image of four spots, two of which display selectivity for Lassa VLPs (in red-top 70:30 DMPAm:HA, bottom 70:30 DMPAm:HEMA) and two that display selectivity for Rubella VLPs (in green-top 70:30 pEGDA:SEMA, bottom 70:30 pEGDA:tBAEMA).

VLP would thus have only a slight negative charge at the incubation conditions and would not be expected to bind preferentially to only positively charged monomers. However, charge heterogeneities on the VLPs could nevertheless result in regions of more dense charge or of H-bond acceptor/donor interactions, which could still allow spatially matched charge-charge and H-bonding interactions with an appropriately matched surface. The highest selectivity index for Rubella over Lassa was found to be from the 


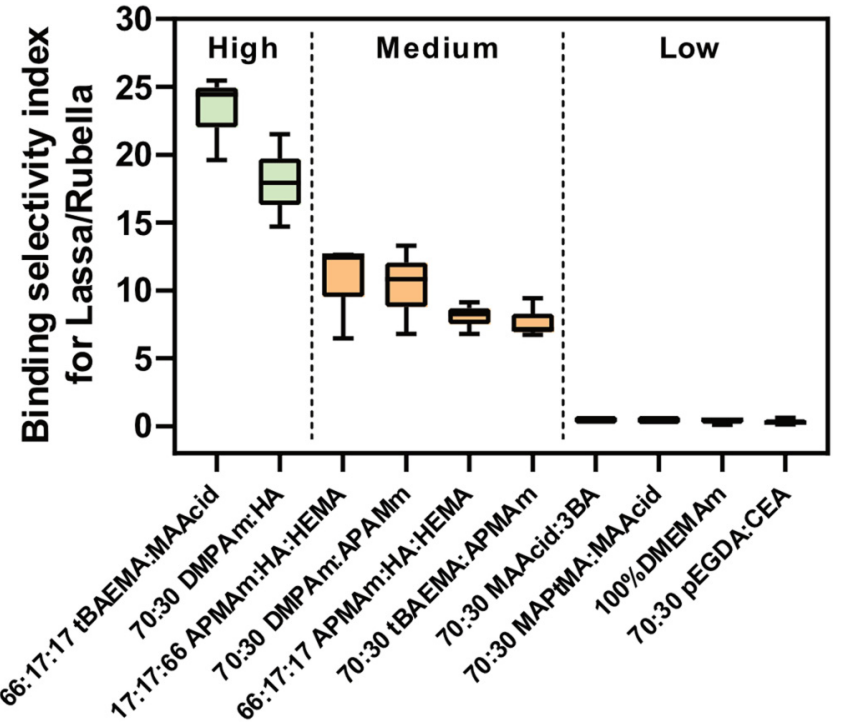

FIG. 2. Binding selectivity indices for Lassa/Rubella for a selection of VLP binding copolymers grouped by statistically significant selectivity for Lassa VLP over Rubella $(p<0.05)$. Monomer compositions on the $x$-axis refer to weight ratios. Data were calculated from the fluorescence images depicted in Fig. 1. Background was subtracted from the intensity values (per pixel) before the values were ratioed. Error given is the standard deviation from three technical replicates on one array slide. Groupings depicted are taken from the Tukey HSD test carried out at a 95\% confidence interval and show representative groups from the dataset.

70:30 pEGDA:CEA comonomer combination (although it should be noted that the standard deviation from the three repeats is relatively high) (Fig. 2, Fig. S4, and Table S3). ${ }^{21}$ The top Rubella binders typically include more acidic and neutral polymers rather than the strongly cationic polymers, which were observed to be more selective for Lassa VLPs. It is expected that the E1 protein would play the largest role in the surface charge of Rubella VLP and this has an isoelectric point (pI) of 6.5. ${ }^{20}$ Thus, it was apparent that simple charge-charge interactions were not predominant for VLP binding at surfaces, which may have arisen due to the surface presentation of the viral spike glycoproteins constrained at the VLP surface compared to the recombinantly expressed and purified proteins themselves.

Further experiments are required to fully understand the mechanisms by which the VLPs interacted with the different polymer surfaces and to provide quantitative data, yet it is clear here that the developed method is able to identify multifaceted candidate materials that interact with VLPs in a rapid and experimentally simple fashion. The ability to screen multiple combinations simultaneously for their binding affinity, therefore, has the potential to identify new and perhaps unanticipated candidate materials suitable for more selective binding and inactivation of viruses. Assessment of absolute binding affinity would require the construction of adsorption isotherms using a range of virion concentrations, ideally complemented with the absolute quantification of the viral load.

\section{CONCLUSION}

In summary, we have reported the development of a highthroughput copolymer microarray system, which enabled rapid identification of copolymers with competitive adsorption of fluorescently tagged VLPs. While more investigation is required to understand fully the mode of binding of the VLPs to the different polymer surfaces, there were clear differences in selectivity observed during the comparative binding experiments, which could be exploited when developing novel materials. As the method was exemplified using virus-like particles, which mimic the structure of the native virus but without the viral genome, this study marks a starting point to study material-virion interactions. Determination of absolute viral load, more complex media models of real situations, and measurement of inactivation of live virus using scaled up analysis in high containment facilities are the next steps. Novel materials identified from such an approach could be exploited in applications including the downstream purification of vaccine components or the more clinically informed design of personal protective equipment.

\section{ACKNOWLEDGEMENTS}

This research was funded by the EMD Millipore Corporation, the life sciences division of Merck KGaA Darmstadt, Germany. This research was also funded by the Department of Health and Social Care using UK Aid funding and is managed by the Engineering and Physical Sciences Research Council (EPSRC, Grant No. EP/R013764/1). This research was also funded through the Royal Society through a Wolfson Research Merit Award (No. WM150086) to C.A. and supported by the Engineering and Physical Sciences Research Council (Grant No. EP/N006615/1]. T.S. received funding from the Deutsche Forschungsgemeinschaft (DFG, German Research Foundation)-Project No. 197785619/ SFB1021. J.D. received funding from the NIHR Nottingham Biomedical Research Centre. The views expressed are those of the authors and not necessarily those of the NHS, the NIHR, or the Department of Health and Social Care.

\section{DATA AVAILABILITY}

The data that support the findings of this study are openly available in Nottingham Research Data Management Repository at https://www.nottingham.ac.uk/it-services/research/applications/ data.aspx.

\section{REFERENCES}

${ }^{\mathbf{1}}$ S. Aydin, Peptides 72, 4 (2015).

${ }^{2}$ X. Zeng, Z. Shen, and R. Mernaugh, Anal. Bioanal. Chem. 402, 3027 (2012).

${ }^{3}$ H. Rosilo, J. R. McKee, E. Kontturi, T. Koho, V. P. Hytönen, O. Ikkala, and M. A. Kostiainen, Nanoscale 6, 11871 (2014).

${ }^{4}$ S. Ma, Y. Li, C. Ma, Y. Wang, J. Ou, and M. Ye, Adv. Mater. 31, 1902023 (2019).

${ }^{5}$ T. M. Przybycien, N. S. Pujar, and L. M. Steele, Curr. Opin. Biotechnol. 15, 469 (2004).

${ }^{6}$ W. J. Brittain, T. Brandsetter, O. Prucker, and J. Rühe, ACS Appl. Mater. Interfaces 11, 39397 (2019).

${ }^{7}$ A. D. Celiz et al., Biomater. Sci. 2, 1604 (2014). 
${ }^{8}$ A. Conde-González, D. Dutta, R. Wallace, A. Callanan, and M. Bradley, Mater. Sci. Eng. C 108, 110489 (2020).

${ }^{9}$ A. L. Hook, D. G. Anderson, R. Langer, P. Williams, M. C. Davies, and M. R. Alexander, Biomaterials 31, 187 (2010).

${ }^{10}$ E. Chiodi, L. Sola, D. Brambilla, M. Cretich, A. M. Marn, M. S. Ünlü, and M. Chiari, Anal. Bioanal. Chem. 412, 3477 (2020).

${ }^{11}$ A. L. Hook, C.-Y. Chang, J. Yang, S. Atkinson, R. Langer, D. G. Anderson, M. C. Davies, P. Williams, and M. R. Alexander, Adv. Mater. 25, 2542 (2013).

${ }^{12}$ S. Eickelmann, A. Tsouka, J. Heidepriem, G. Paris, J. Zhang, V. Molinari, M. Mende, and F. F. Loeffler, Adv. Mater. Technol. 4, 1900503 (2019).

${ }^{13}$ A. L. Hook et al., Nat. Biotechnol. 30, 868 (2012).

${ }^{14}$ D. G. Anderson, S. Levenberg, and R. Langer, Nat. Biotechnol. 22, 863 (2004).

${ }^{15}$ A. L. Hook et al., J. Vis. Exp. 59, e3636 (2012).
${ }^{16}$ J. Gong, S. Venkateswaran, M. G. Tanner, J. M. Stone, and M. Bradley, ACS Comb. Sci. 21, 417 (2019).

${ }^{17}$ C. R. E. Duffy, R. Zhang, S.-E. How, A. Lilienkampf, G. Tourniaire, W. Hu, C. C. West, P. de Sousa, and M. Bradley, Biomater. Sci. 2, 1683 (2014).

${ }^{18}$ K. Schlie, A. Maisa, F. Freiberg, A. Groseth, T. Strecker, and W. Garten, J. Virol. 84, 3178 (2010).

${ }^{19}$ S. Verma, S. Yadav, and A. Kumar, Adv. Biomed. Res. 4, 201 (2015).

${ }^{20} \mathrm{M}$. N. Waxham and J. S. Wolinsky, Rev. Infect. Dis. 7, S133 (1985).

${ }^{21}$ See supplementary material at https://doi.org/10.1116/6.0000586 for information with regards to array fabrication and characterization, virus like particle preparation, statistical analysis information, and supporting data (phase contrast imaging, ToF-SIMS, and fluorescence). 